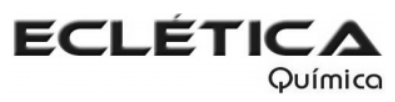

www.scielo.br/eq

www.ecletica.iq.unesp.br

Volume 33, número 4, 2008

\title{
Degradação hidrolítica e fotoquímica da amoxicilina na presença de $\beta$-ciclodextrina
}

\author{
R. A. Bariccatti*, C. Silva, M. L. Souza, C. A. Lindino, M. F. Rosa \\ Centro de Engenharias e Ciências Exatas - Universidade Estadual do Oeste do Paraná - CEP- \\ 85903-000 - Toledo - Pr - Brasil \\ *reinaldo0207@brturbo.com.br
}

Resumo: Este trabalho tem como propósito monitorar a degradação do fármaco amoxicilina na presença e ausência de $\beta$-ciclodextrina através de técnicas espectroscópicas. Para isto, foi acompanhada a hidrólise do fármaco protegido da luz por cerca de 400 horas. Os resultados obtidos indicam que, inicialmente, a ciclodextrina não altera a hidrólise da amoxicilina, entretanto, após 250 horas de monitoramento há um aumento acentuado na hidrólise da amoxicilina quando presente a ciclodextrina. Ao irradiar a amostra com radiação UV, verificamos que as soluções contendo $\beta$-ciclodextrina sofrem uma fototransformação mais lenta $(26,8 \%)$ que as soluções sem $\beta$-ciclodextrina..

Palavras-chave: Amoxicilina; $\beta$-ciclodextrina; fotodegradação.

\section{Introdução}

As ciclodextrinas (CDs) são maltooligossacarídeos cíclicos constituídos por um número variável de unidades de glucose (geralmente de 6 a 8), unidos por ligações $\alpha-1,4$. As mais comuns são: $\alpha-C D$ (ciclohexamilose), $\beta-C D$ (cicloheptamilose) e $\gamma$-CD (ciclooctamilose). As CDs têm forma de cone truncado com uma cavidade interna cujo tamanho e a forma são determinados pelo número de unidades de glucose[1]. Esse interior é relativamente apolar, comparado com a água, formando complexos de inclusão com várias substâncias orgânicas[2].

A inclusão em CD pode aumentar a estabilidade da molécula hospedeira inviabilizando mecanismo de quebra molecular, por exemplo, frente ao calor, levando a uma redução na volatilidade ou aumento da resistência térmica. Para princípios ativos relativamente insolúveis em água, a inclusão pode melhorar sua solubilidade ou sua cinética de dissolução. Uma elevada constante de estabilidade do complexo favorece a passagem do princípio ativo através da membrana celular, aumentando sua atividade biológica[3]. As CDs são capazes de complexar e de estabilizar um largo espectro de substâncias, essa característica, associada às outras propriedades das CDs já citadas, faz delas uma grande atração para um variado número de aplicações industriais. As vantagens em potencial do uso e da disponibilidade das CDs têm tido papel decisivo no crescente interesse pelas mesmas, podendo ser usadas em fármacos, alimentos, cosméticos, etc, para estabilizar frente à radiação UV[4], temperatura, oxidação e hidrólise; aumentar a solubilidade em água[5]; mudanças de propriedades reológicas; liberação controlada de ingredientes ativos; mascarar odores e gostos desagradáveis; aumentar a biodisponibilidade; modelo de enzi- 
ma[6]; extração seletiva, adsorção de poluentes[7] e reatores moleculares[8].

A amoxicilina (Am) é um antibiótico semisintético com estrutura básica da penicilina $\mathrm{G}$ que atua em bactérias gram positivas e gram negativas. Formam vários complexos com íons metálicos[9], tais como $\mathrm{Fe}(\mathrm{II}), \mathrm{Ni}(\mathrm{II}), \mathrm{Zn}$ (II) etc. Estes íons atuam como catalisadores, degradando a amoxicilina por meio de um processo de solvólise. Ela também é rapidamente degradada sob condições ácidas por causa do anel $\beta$-lactâmico que é sucessível a degradação hidrolítica quando o $\mathrm{pH}$ desvia significativamente do ponto isoelétrico $(\mathrm{pH} 4,8)$. Devido portanto a esta baixa estabilidade propõese estudar o efeito da adição de ciclodextrina na degradação da amoxicilina em meio aquoso protegido da luz e sob irradiação UV.

\section{Procedimento}

Foram preparadas soluções no $\mathrm{pH}$ 7,3 utilizando soluções de $\mathrm{Na}_{2} \mathrm{HPO}_{4}$ e $\mathrm{KH}_{2} \mathrm{PO}_{4} 0,1 \mathrm{~mol} / \mathrm{L}$ segundo Morita[10].A amoxicilina foi obtida junto à empresa farmacêutica Prati-Donadutti.

Para os estudos de concentração foram preparadas soluções mãe de amoxicilina em água destilada e por diluição obteve-se a concentração desejada.

No estudo de fotodegradação foi preparada uma solução conforme procedimento anterior, sendo que, antes de se completar o volume com água foi adicionado um volume de solução aquosa de ciclodextrina, que em seguida foi exposta à irradiação proveniente de uma lâmpada de mercúrio de $80 \mathrm{~W}$ de potência.

\section{Resultados e Discussão}

Inicialmente, alterou-se a concentração da amoxicilina para se determinar o coeficiente de absorção molar e verificar a formação de agregados. Não foram verificadas alterações no formato e posição das bandas de absorção com a variação na concentração do fármaco, indicando que na faixa de concentração de interesse $\left(1,0 \times 10^{-5}\right.$ até $4,0 \times 10^{-4} \mathrm{~mol} \mathrm{~L}^{-1}$ ) não houve formação de agregados ou excímeros do fármaco e sim apenas aumento da intensidade devido ao efeito da concentração (Lei de Beer), as absorbâncias molares em 228 e $273 \mathrm{~nm}$ são $7.440 \pm 180$ e $870 \pm 11 \mathrm{~mol}^{-}$ ${ }^{1}$.L.cm-1 (Figura 1), respectivamente.

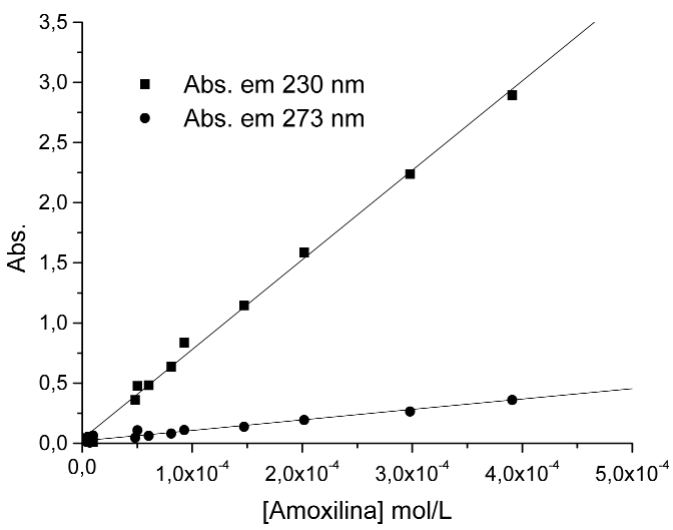

Figura 1. Gráfico com as absorbâncias, em $230 \mathrm{~nm}$ (घ) e $273 \mathrm{~nm}(\mathbf{O})$, das soluções de amoxicilina em várias concentrações.

Os resultados de estabilidade da amoxicilina, na ausência de irradiação UV, em função do tempo, em solução aquosa são apresentados na Figura 2. Observa-se o surgimento de uma nova banda de absorção centrada em 350nm que aumenta em função do tempo.

Os estudos da alteração espectral, no comprimento de onda de $350 \mathrm{~nm}$, em função do tempo (de 15 a 20 dias) foram feitos em triplicata e em

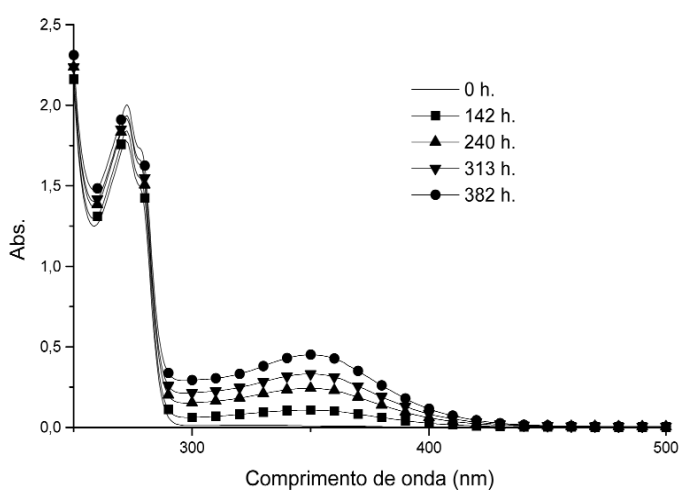

Figura 2. Gráfico com as absorbâncias da solução de amoxicilina pura em função do tempo (horas), $[\mathrm{Am}]=2,0 \times 10^{-3} \mathrm{~mol} \mathrm{~L}^{-1}$. 
três períodos distintos sem o controle de temperatura, representando o que normalmente ocorreria ao fármaco caso o mesmo fosse estocada em solução aquosa na prateleira e são mostrados na Figura 3. Observa-se um aumento inicial que segue um comportamento linear nas 150 horas iniciais com a equação da reta de $\mathrm{y}=0,34 \mathrm{t}$. Após este período temos um comportamento linear com equação de $y=-175+1,10 t$, onde y é a relação entre a absorbância e a concentração da amoxicilina e $\mathrm{t}$ o tempo em horas.

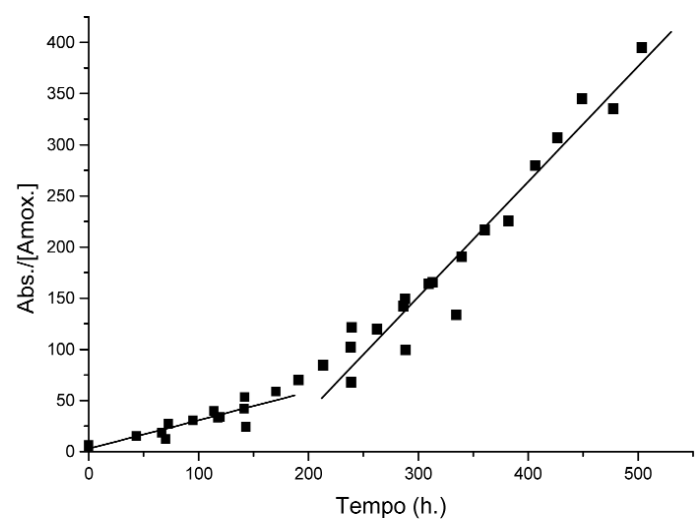

Figura 3. Gráfico com as absorbâncias em $350 \mathrm{~nm}$, normalizada pela concentração das soluções de amoxicilina puras em função do tempo (horas), realizadas em triplicata $(2,0 ; 2,1 \mathrm{e}$ $\left.3,6.10^{-3} \mathrm{~mol} \mathrm{~L}^{-1}\right)$.

Navarro[11] estudou o efeito da temperatura na solvólise da amoxicilina catalisada por íons metálicos em metanol e verificou o surgimento de um produto fluorescente com máximo de excitação em 362nm e de emissão em 442nm que sofre catálise pelos íons $\mathrm{Cd}^{2+}, \mathrm{Zn}^{2+}$ e $\mathrm{Co}^{2+}$. Segundo este autor o íon que fornece a menor energia de ativação é o íon $\mathrm{Zn}^{2+}$, cujo valor é $34,6 \mathrm{~kJ} \mathrm{~mol}^{-1}$, e na ausência deste íon a energia de ativação é de 114,6 $\mathrm{kJ} \mathrm{mol}^{-1}$. Em outro artigo o mesmo autor[12] estuda a formação de complexo de 1:1 e 2:1 de (ampilicilina/cádmio) e a constante de velocidade de degradação do complexo 1:1. Além disto, o autor estuda as alterações estruturais por meio de RMN e verifica o surgimento de grupamento éster e quebra do anel $\beta$-lactâmico. Pelo espectro de massas de impacto de elétron verifica a formação de um produto que é a união de dois fragmentos da molécula de ampilicilina. Estudos realizados por Nägele e Moritz[13] fornecem resultados semelhantes do espectro de massas para a degradação da amoxicilina em meio ácido e em dimetil sulfóxido. Segundo os autores, inicialmente, há formação de dois produtos, ácido penicilóico amoxicilínico e 4hidroxifeniglil amoxicilina. O ácido penicilóico amoxicilínico origina mais duas outras substâncias, ácido penilóico amoxicilínico e a amoxicilina dicetopiperazina. Segundo estes autores, a solvólise da amoxicilina leva à formação de um éster ou ácido da amoxicilina, dependendo do solvente, e a formação de um produto que se origina de dois fragmentos da amoxicilina.

Assim, as modificações espectrais observadas nas Figuras 2 e 3 podem ser atribuídas a estas modificações estruturais, onde há formação de um produto final que absorve em $350 \mathrm{~nm}$.

$O$ efeito da adição de $\beta$-CD na solução de amoxicilina é mostrado na Figura 4. Segundo os resultados a ciclodextrina causa um aumento considerável na absorbância após um período de incubação de cerca de 250 horas. Yannakopoulou[14], estudando a hidrólise da ampilicilina e amoxicilina, não verificou a alteração da velocidade de hidrólise dos antibióticos na presença de ciclodextrina. Aki[15], em estudos calorimétricos, não verificou alteração na velocidade de hidrólise na presença de derivados da ciclodextrina em pH 6, mas em pH 2 verificou alteração significativa na velocidade de hidrólise. Os resultados mostrados na Figura 4 concordam com os resultados dos ambos os autores, já que estes estudaram a hidrólise em uma faixa de tempo inferior à observada neste trabalho. Entretanto, o forte aumento observado em $350 \mathrm{~nm}$ após um período de cerca de $250 \mathrm{~h}$. não foi relatado por estes autores e está sendo associado a uma autocatálise ácida também observada para a solução de amoxicilina pura (Figura 3), na qual, a $\beta$-CD favorece este processo acentuadamente.

A estrutura da amoxicilina permite o encapsulamento por ambos os lados da molécula, o que poderia influenciar a degradação de cada um dos cromóforos de forma diferente. Assim, caso o encapsulamento ocorresse apenas no anel benzênico este seria protegido da degradação ao passo que o segundo cromóforo não o seria, bem como, o 


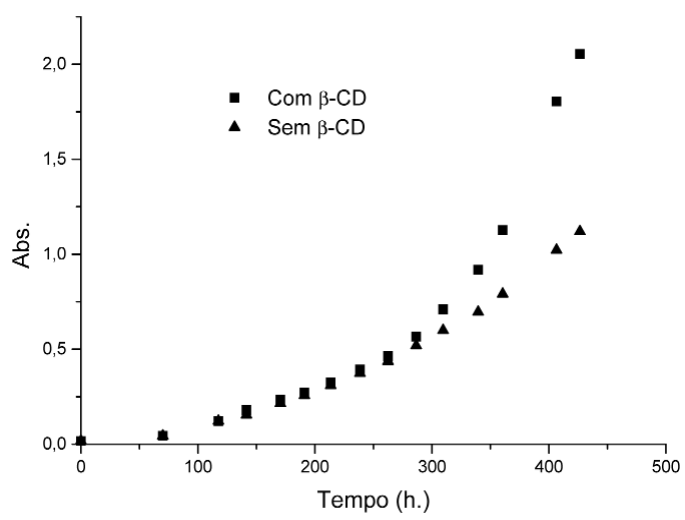

Figura 4. Gráfico com a absorbância em 350nm das soluções de amoxicilina sem $\beta$-CD $(\boldsymbol{\Delta})$ e amocixilina com $\beta-\mathrm{CD}(\boldsymbol{\square})$ em função do tempo (horas).

oposto também poderia ocorrer. As transições associada ao anel benzênico $\left(\pi-\pi^{*}\right)$ fornece bandas mais intensas e em comprimento próximo de 200nm, já a carbonila ocorrem em comprimento de onda superior a $250 \mathrm{~nm}$ e são bandas de baixa intensidades, assim, monitorando os diferentes comprimentos de onda podemos verificar a transformação de diferentes cromóforos.

A Figura 5 apresenta o efeito da radiação UV nas soluções de amoxicilina e amoxicilina/ $\beta$ $\mathrm{CD}$, dentre outras alterações, observa-se mudanças na intensidade de absorção nas regiões de 195 e $225 \mathrm{~nm}$. Para a região de $195 \mathrm{~nm}$, ocorre uma redução contínua da absorção para as soluções com amoxicilina e amoxicilina/ $\beta-C D$, esta redução foi de $45,2 \%$ na solução de amoxicilina (absorbância de 1,99-1,09) e na solução amoxicilina/ $\beta$-CD foi de 36,3\% (absorbância de 2,04 ?1,30), após 120 minutos de irradiação. Em valores absolutos, houve uma redução na degradação do cromóforo que absorve em $195 \mathrm{~nm}$ de $9 \%$. Possivelmente, nesta região espectral ocorre a absorção de luz dos derivados benzênicos que são encapsulados e estabilizados pela ciclodextrina.

Para a região de 225nm (Figura 5) observa-se um aumento na absorbância nos primeiros 30 minutos de irradiação para em seguida ocorrer uma redução contínua da absorbância desta região, tanto na solução de amoxicilina e amoxicilina/ $\beta-C D$. Para registrar este aumento de absorbância foi monitorado $\mathrm{o}$ aumento da

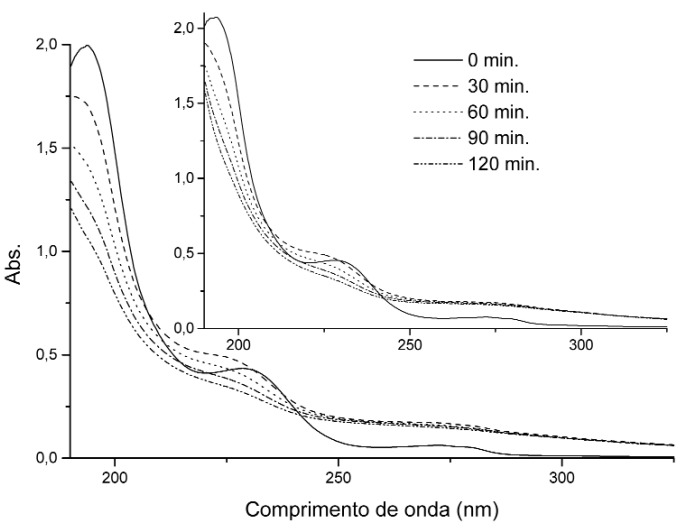

Figura 5. Gráficos mostrando as absorbâncias das soluções de amoxicilina com diferentes tempos de irradiação. Gráfico principal, soluções contendo amoxicilina sem $\beta$-CD e gráfico inserido soluções de amoxicilina com $\beta$-CD, $[\mathrm{Am}]=5,0 \times 10^{-5} \mathrm{~mol} \mathrm{~L}^{-1}$ e $[\beta-C D]=4,0 \times 10^{-3} \mathrm{~mol} \mathrm{~L}^{-1}$.

absorbância em $228 \mathrm{~nm}$ com irradiação de $3 \mathrm{em}$ 3 minutos durante 21 minutos iniciais, este resultados são apresentados na Figura 6. As retas ajustadas para estes valores possuem inclinações diferentes, indicando diferentes velocidades de formação desta banda. A inclinação da reta para a

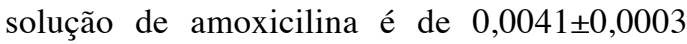
unidades de absorbância por minuto, para a solução de amoxicilina/ $\beta$-CD a inclinação é de $0,0030 \pm 0,0001$ unidades de absorbância por minuto, ou seja, há uma redução na velocidade de

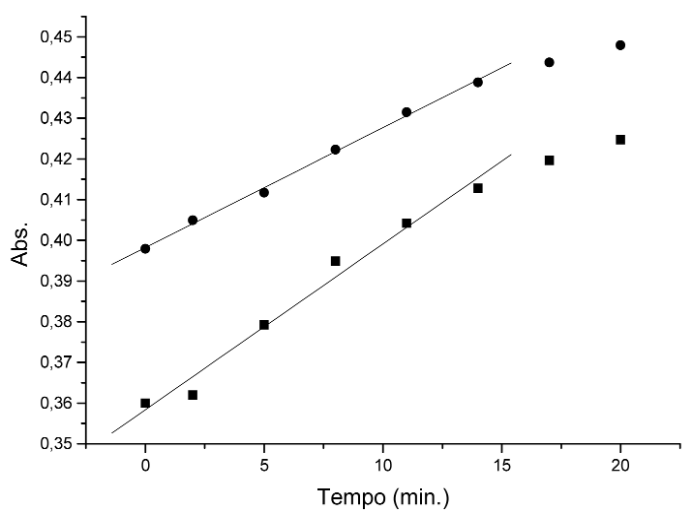

Figura 6. Gráfico com a absorbância em 228nm da solução de amoxicilina sem $\beta$-CD (ם) e com $\beta$-CD (O), [Amox. $]=9,8 \times 10^{-5} \mathrm{~mol} \mathrm{~L}^{-1}$ e $[\beta-$ $\mathrm{CD}]=4,0 \times 10^{-3} \mathrm{~mol} \mathrm{~L}^{-1}$. 
formação desta banda de cerca de $26,8 \%$, quando comparada com a solução sem $\beta$-CD. Ou seja, ao irradiar a amoxicilina ocorre uma fototransformação nos primeiros 21 minutos que induz um aumento de absorbância em $228 \mathrm{~nm}$, entretanto, este aumento ocorre de maneira mais lenta na solução contendo $\beta$-CD.

\section{Conclusão} distintos.

Nestes estudos observaram-se dois efeitos

$\mathrm{Na}$ ausência de irradiação a amoxicilina não é estabilizada pela $\beta$-CD, não alterando o comportamento da absorbância em 350 nm num período de 250 horas, após este período verifi- cou-se um aumento da absorbância em 350nm que é intensificado pela adição de $\beta$-CD.

$\mathrm{Na}$ presença de irradiação UV, verificouse que a $\beta$-CD causa uma estabilização na amoxicilina de aproximadamente $26,8 \%$, reduzindo a velocidade de transformação desta substância em um fotoproduto, que possui uma banda mais intensa em 228nm. Para a parte aromática da amoxicilina ocorre um processo de degradação fotoquímica contínua, reduzindo sua absorbância na região de $190 \mathrm{~nm}$, entretanto, a $\beta$-CD possui um efeito estabilizador neste cromóforo na ordem de $9 \%$.

Recebido em 23 de maio de 2008

Aceito em 05 de novembro de 2008

R. A. Bariccatti, C. Silva, M. L. Souza, C. A. Lindino, M. F. Rosa. Hydrolytic and photochemistry degradation of the amoxicillin in $\beta$-cyclodextrin.

\begin{abstract}
This work has like purpose monitors the degradation of the drug amoxicillin in the presence and absence of $\beta$-cyclodextrin, through techniques spectroscopy. For this, there was accompanied the hydrolysis of the drug protected of the light for around 400 hours. The results indicate that, initially, the cyclodextrin does not alter the hydrolysis of the amoxicillin, however, after 250 hours there is an increase of the hydrolysis of the amoxicillin when present at cyclodextrin. Another variable was the irradiation of the sample with radiation in the region of the UV, we see that the solutions containing $\beta$ cyclodextrin suffer a slower phototransformation $(26,8 \%)$ than the solutions without $\beta$-cyclodextrin, when irradiated by UV radiation.
\end{abstract}

Keywords: Amoxicillin; $\beta$-cyclodextrin; photodegradation.

\section{Referências}

[1] Y. D. Lee, H. S. Kim, Biotechnology and Bioengineering 37 (1991) 795-801.

[2] Szejtli, J.; Cyclodextrin Technology, Kluwer Academic Publishers, 1988.

[3] D. Duchêne, C. Vaution, F. Glomot, Drug Dev. Ind. Pharm. 12(11-13) (1986) 2193-2215.

[4] R. A. Bariccatti, et al., Acta Scientiarum Health Science 27 (2005) 171-175.

[5] K. Uekama, Journal Inclusion Phenomena and Macrocyclic Chemistry 44 (2002) 3-7.

[6] M. L. Bender, Journal American Chemistry Society 89 (1967) 3242-3253.

[7] G. Crini, et al., Separation and Purification Tecnology 53
(2007) 97-110.

[8] L. Barr, et al., J. Inclusion Phenomena and Macrocyclic Chemistry 50 (2004) 19-24.

[9] M. A. Zayed, S. M. Abdallah, Spectrochimica Acta Part A 61 (2005) 2231-2238.

[10] R. M. V. Assumpção, T. Morita, Manual de soluções, reagentes e solventes, Edgard Blucher, São Paulo, 1968.

[11] P. G. Navarro, et al., Analyst 123 (1998) 2263-2266.

[12] P. G. Navarro, et al., Talanta 46 (1998) 101-109.

[13] E. Nägele, R. Moritz, J. Am. Mass. Spectrom. 16 (2005) 1670-1676.

[14] K. Yannakopoulou, et al., Organic \& Biomolecular Chemistry 4 (2006) 1297-1304.

[15] H. Aki, et al., J. Thermal Analysis and Calorimetry 77 (2004) 423-435. 